ARTICLE

Received 1 Apr 2010 | Accepted 9 Sep 2010 | Published 5 Oct 2010

DOI: $10.1038 /$ ncomms1089

\title{
A systems approach towards the stoichiometry- controlled hetero-assembly of nanoparticles
}

Yong Wang', Gang Chen', Miaoxin Yang', Georg Silber', Shuangxi Xing', Li Huey Tan', Feng Wang², Yuhua Feng' ${ }^{1}$ Xiaogang Liu², Shuzhou Li ${ }^{3}$ \& Hongyu Chen ${ }^{1}$

A central theme in nanotechnology is to advance the fundamental understanding of nanoscale component assembly, thereby allowing rational structural design that may lead to materials with novel properties and functions. Nanoparticles (NPs) are often regarded as 'artificial atoms', but their 'reactions' are not readily controllable. Here, we demonstrate a complete nanoreaction system whereby colloidal NPs are rationally assembled and purified. Two types of functionalized gold NPs ( $A$ and $B$ ) are bonded to give specific products $A B, A B_{2}, A B_{3}$ and $A B_{4}$. The stoichiometry control is realized by fine-tuning the charge repulsion among the $B-N P$ s. The products are protected by a polymer, which allows their isolation in high purity. The integration of hetero-assembly, stoichiometry control, protection scheme and separation method may provide a scalable way to fabricate sophisticated nanostructures.

\footnotetext{
1 Division of Chemistry and Biological Chemistry, School of Physical and Mathematical Sciences, Nanyang Technological University, Singapore 637371, Singapore. ${ }^{2}$ Department of Chemistry, National University of Singapore, Singapore 117543, Singapore. ${ }^{3}$ Division of Materials Science, School of Materials Science and Engineering, Nanyang Technological University, Singapore 639798, Singapore. Correspondence and requests for materials should be addressed to H.C. (email: hongyuchen@ntu.edu.sg).
} 
C olloidal growth of nanoparticles (NPs) has been a major focus in nanoscience over the past decades. It provides a scalable and versatile approach to control the size and morphology of NPs, which are critical for their properties. NPs with simple geometries, such as spheres, rods, cubes and triangular prisms, have been prepared with excellent control ${ }^{1-5}$. In contrast, colloidal assembly of NPs has been less studied, although it could conceivably offer novel synthetic controls for multi-component nanoclusters. The key challenge is to develop general methodologies to assemble colloidal nano-objects with precise stoichiometry. Previously, homo-aggregates such as dimers and trimers were prepared by exploiting either random aggregation ${ }^{6-8}$, steric effects ${ }^{9-12}$ or specific control over surface ligands (for example, divalent NPs) ${ }^{13-16}$. Compared with these 'homo-coupling' nanoreactions, controlling stoichiometry in the 'cross-coupling' of NPs presents different and arguably greater challenges. It was only achieved through the brilliant design and pre-fabrication of NPs with anisotropic surface functionalities (for example, NP with a single DNA molecule) $)^{17-22}$.

In a sense, the assembly of NPs in a colloidal solution is analogous to molecular reactions. Rationally controlled organic syntheses, completed with coupling, protection and separation schemes, have given rise to complex molecules with precise structural control. From this perspective, a complete nanoreaction system emulating the concepts and strategies of molecular reactions may offer a systematic approach to sophisticated nanostructures.

A major problem in assembling NPs is the preservation of the product nanoclusters against both disintegration and aggregation, which is often exacerbated by ligand dissociation and exchange over long periods of time. We have recently demonstrated that encapsulation of nanoclusters in polystyrene-block-poly(acrylic acid) (PSPAA) micelles is a way to overcome these problems and affords sufficient stability for the nanoclusters to be separated using concentrated $\mathrm{CsCl}$ solutions $\mathrm{s}^{6,23,24}$.

Here, we combine the protection and purification schemes with the hetero-assembly of NPs, making it possible to recover pure nanoclusters with long-term stability. The stoichiometry of the assemblies was controlled by modulating the charge repulsion among the NPs. The systems approach led to nanoreactions with new structural control. We show that this complete nanoreaction system is an ideal platform for studying the interactions of NPs, just as the studies of molecular reactions provide insight to the chemical nature of atoms.

\section{Results}

Assembly of $\mathbf{A B}_{n}$ nanoclusters. Two types of NPs were prepared as the basic reactants: group A was gold NPs (AuNPs) coated with a tetrathiol molecule, 1 (Fig. 1a, giving A-NPs), whereas group B was citrate-stabilized AuNPs (B-NPs; Fig. 2a). To mimic molecular reactions, it is critical that these two types of NPs could react with each other, but do not form aggregates of their own. The thiol groups of $\mathbf{1}$ allowed the ligand to firmly anchor on AuNP while presenting extra thiol groups that could bond to additional AuNPs (that is,

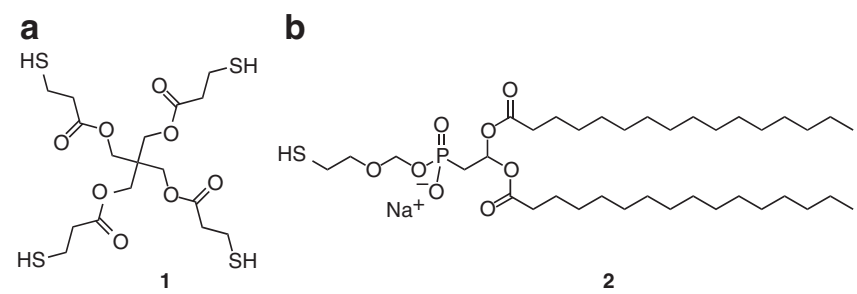

Figure 1 | Structure of the two ligands used in this study. (a) A tetrathiol ligand, pentaerythritol tetrakis(3-mercapto-propionate) (1). (b) A thiol-ended hydrophobic ligand, 2-dipalmitoyl-sn-glycero-3phosphothioethanol (sodium salt) (2). forming AuNP-1-AuNP bridges through the Au-S interactions). Hence, the A-NPs have to be completely covered with $\mathbf{1}$, so that they would not provide exposed Au surface for bonding among themselves. These NPs were, thus, synthesized by treating citrate-AuNPs with excess $\mathbf{1}$, and the free ligands were then removed by centrifugation. The purified A-NPs were relatively stable in dimethylformamide (DMF), but they were used shortly after the synthesis. In the absence of excess 1, the A-NPs could undergo partial ligand dissociation, which will lead to aggregation. Finally, the stoichiometry of the NP assemblies has to be controlled. Without restrictions, multiple B-NPs could bond to each A-NP and vise versa, leading to extensive and uncontrolled aggregation of the two reactants (Supplementary Figs S1 and S2). Our strategy here is to treat A-NPs with excess $\mathrm{B}-\mathrm{NPs}$, and to control the stoichiometry of the product $\mathrm{AB}_{n}$ by finetuning the repulsion between the B-NPs.

In a colloidal reaction, the purified A-NPs in DMF $(100 \mu \mathrm{l}$, $d_{\mathrm{A}}=18 \mathrm{~nm}$ ) was slowly added to an aqueous solution containing large excess of B-NPs $\left(200 \mu \mathrm{l}, d_{\mathrm{B}}=18 \mathrm{~nm}\right.$, and final $[\mathrm{A}]:[\mathrm{B}]=1: 16$; see details in Supplementary Methods). The random collision between $\mathrm{A}$ - and $\mathrm{B}-\mathrm{NPs}$ gives $\mathrm{AB}_{n}$ nanoclusters. However, the product solution cannot be directly characterized by transmission electron microscopy (TEM), as any drying or purification process would lead to significant aggregation of the solution species. Hence, both the product $\mathrm{AB}_{n}$ and the remaining B-NPs were protected by PSPAA encapsulation (Fig. 2b-e), which basically involved the functionalization of the B-NP surface by a hydrophobic ligand (2, Fig. 1b), followed by PSPAA self-assembly ${ }^{6,25,26}$. Ligand 2 cannot replace the hydrophilic ligand $\mathbf{1}$ on the surface of A-NPs, which was thus not encapsulated. The resulting $\mathrm{AB}_{n} @ P S P A A$ and B@PSPAA were readily isolated from the preparative solution by centrifugation, and then they were re-dispersed in water. With the polymer protection, the nanoclusters are stable against dissociation and aggregation. This allowed detailed product analysis of the nanoreactions by TEM characterization, giving a window for mechanistic insights.

Figure $2 c$ shows the TEM image of a sample that contained trimeric nanoclusters, where the polymer shells only attached to the AuNPs on the ends but not to the central one. The uniformity of this unique polymer coverage (Fig. $2 \mathrm{~g}$ ) indicated that the trimeric clusters were not $\mathrm{B}_{3} @ P S P A A$, but rather (BAB)@PSPAA. This assignment is consistent with the expectations that 2 could replace citrate ions on B-NPs but not the multidentate 1 on A-NPs, and that PSPAA could only attach to regions covered with non-polar and hydrophobic ligands such as 2 (ref. 12). Thus, the nanoreaction was successful in giving the $\mathrm{AB}_{2}$ nanoclusters. The reaction product was surveyed based on the TEM images (Supplementary Fig. S3). As excess B-NPs were used, the yield of the product $\mathrm{AB}_{2}$ (89.4\%, Table 1) was calculated based on the number of A-NPs. The by-products included a few $\mathrm{AB}, \mathrm{AB}_{3}$ and also $\mathrm{A}_{x} \mathrm{~B}_{y}$ nanoclusters that may result from aggregated A-NPs. It was intriguing that the nanoreaction terminated at $\mathrm{AB}_{2}$ despite the presence of excess B-NPs. As a NP in close packing has a coordination number of 12 , there was sufficient space on the $\mathrm{AB}_{2}$ for additional bonding with B-NPs. Obviously, some form of repulsion other than steric hindrance was responsible for the stoichiometry control. As strong electric or magnetic dipoles are unlikely for the large spherical AuNPs (>5 nm) (ref. 27), the most likely candidate is charge repulsion. Moreover, the linear $\mathrm{AB}_{2}$ is reminiscent of the linear aggregates of AuNPs reported earlier ${ }^{28,29}$, where charge and dipole interactions were proposed to be the main factors in the geometric control.

To test this proposal, the colloidal reactions were carried out in solutions with varying ionic strengths (Table 1, Supplementary Figs S4-S8), which are known to modulate the charge repulsion between NPs. Although it was difficult to adjust the ionic strength using a single salt owing to the stability requirements for the reactant NPs, it was clear that the size of $\mathrm{AB}_{n}$ nanoclusters increased with increasing $\left[\mathrm{Na}_{3}\right.$ citrate] or $[\mathrm{NaCl}]$ (Table 1 and Fig. 2). In particular, 
the sole increase of $[\mathrm{NaCl}]$ from 0 to $2.25 \mathrm{mM}$ (entry 5 and 6) changed the major product of the nanoreaction from $\mathrm{AB}_{3}(65.6 \%)$ to $\mathrm{AB}_{4}(64.9 \%)$. In entry 4 of Table $1, \mathrm{NaNO}_{3}$ was shown to have a similar effect as $\mathrm{NaCl}$, indicating that the $\mathrm{Cl}^{-}$ions were not particularly important. Thus, the stoichiometry control of $\mathrm{AB}_{n}$ was probably more relevant to the general ionic strength of the solution than other effects. Clear distance existed between the neighbouring $\mathrm{B}-\mathrm{NPs}$ in all $\mathrm{AB}_{n}$ nanoclusters, leading to linear $\left(\mathrm{AB}_{2}\right)$, triangular $\left(\mathrm{AB}_{3}\right)$ or tetrahedron $\left(\mathrm{AB}_{4}\right)$ geometries. Although the large nanoclusters such as $\mathrm{AB}_{3}$ and $\mathrm{AB}_{4}$ were often completely engulfed by the polymer shell (Fig. 2h,i), their structural uniformity indicated that the hetero-assembly were successful and that the central NPs were all A-NPs. Notably, the $\sim 18 \mathrm{~nm}$ separation of the B-NPs in $\mathrm{AB}_{2}$ is a testament for the strong long-range repulsion during the formation of the nanoclusters. Such a force is most probably charge repulsion. It is hence likely that the increase in ionic strength led to more effective shielding of the surface charges, allowing more B-NPs to bond to each A-NP.

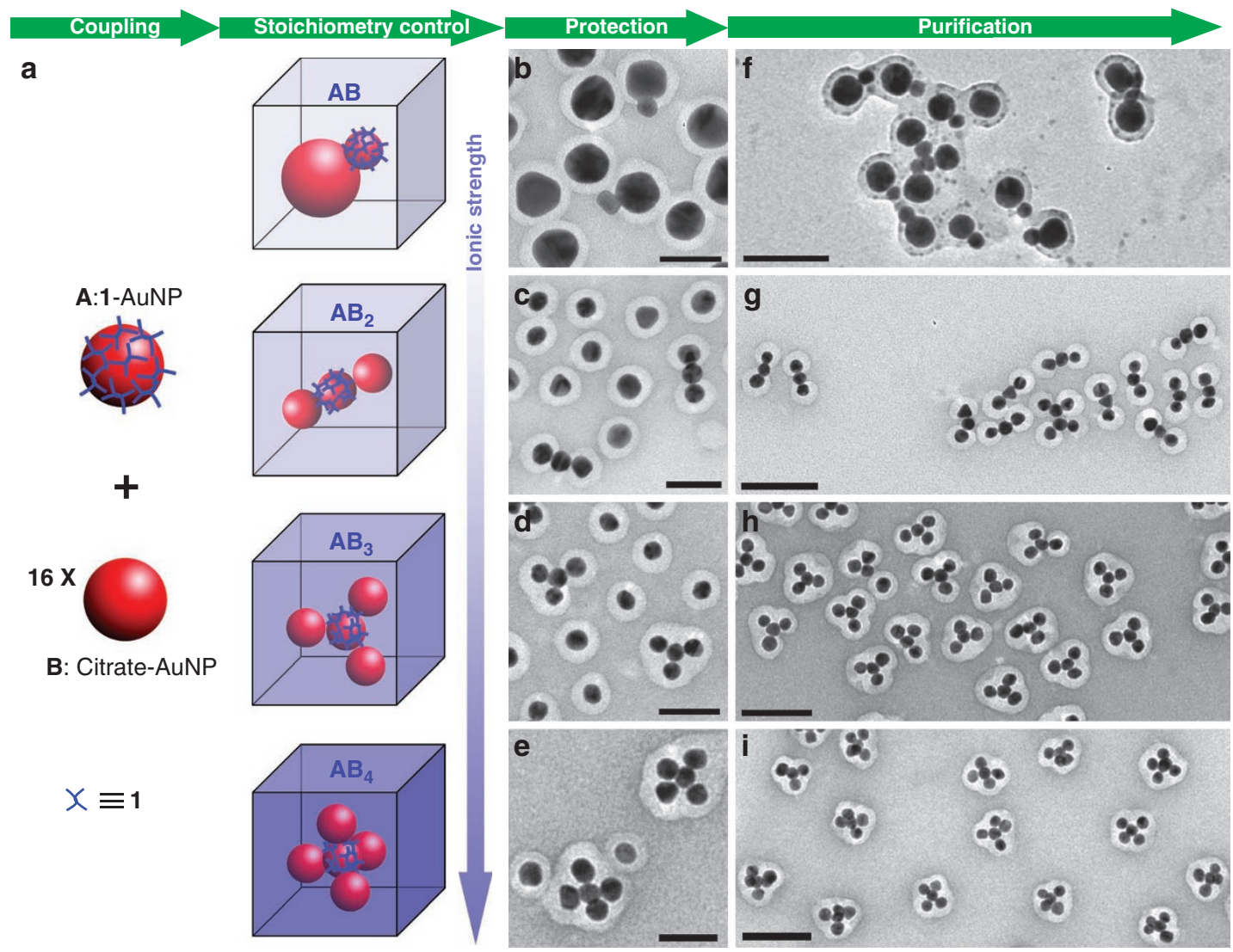

Figure 2 | Stoichiometry-controlled nanoreactions. (a) Schematics showing the syntheses of $A B, A B_{2}, A B_{3}$ and $A B_{4}$ nanoclusters, by tuning the charge repulsion between the B-NPs (through the ionic strength of the reaction media). The intensity of the shade in the boxes is correlated to the ionic strength of the media: from top down, $0.297,0.594,5.04$ and $7.29 \mathrm{mM}$. (b-e) TEM images of the $A B_{n}$ nanoclusters after protection by PSPAA, whereby the nanoclusters were treated with PSPAA and the hydrophobic ligand $\mathbf{2}$. The surface of B-NP is rendered hydrophobic by ligand exchange, on which the PSPAA self-assembles to form a uniform shell. The excess B-NPs are also encapsulated, but the A-NPs are not encapsulated because of persistence of the polar ligand $\mathbf{1}$. See Table 1 for product analyses. (f-i) TEM images of the purified nanoclusters. Scale bars are $50 \mathrm{~nm}$ for $\mathbf{b}-\mathbf{e}$ and $100 \mathrm{~nm}$ for $\mathbf{f - i}$.

Table 1 | Product analyses of the nanoreactions.

\begin{tabular}{|c|c|c|c|c|c|c|c|c|c|}
\hline \multirow[t]{2}{*}{ Entry } & \multirow[t]{2}{*}{$B(\mathbf{n m})$} & \multirow[t]{2}{*}{ I (mM) } & \multicolumn{2}{|c|}{ Salt concentrations } & \multicolumn{4}{|c|}{ Yield $\%$ based on $A^{\star}$} & \multirow[t]{2}{*}{ Isolated purity\%* } \\
\hline & & & $\begin{array}{c}\mathrm{Na}_{3} \text { citrate } \\
(\mu M)\end{array}$ & Additional salt & $\mathbf{A B}$ & $\mathbf{A B}_{2}$ & $\mathbf{A B}_{3}$ & $\mathbf{A B}_{4}$ & \\
\hline 1 & 18 & 0.297 & 49.5 & - & 18.2 & 74.0 & 1.98 & 0 & - \\
\hline 2 & 18 & 0.594 & 98.9 & - & 5.80 & 89.4 & 0.39 & 0 & $\mathrm{AB}_{2}, 87.3^{\dagger}, 85.9$ \\
\hline 3 & 18 & 2.59 & 98.9 & $2.00 \mathrm{mM} \mathrm{NaCl}$ & 1.54 & 62.2 & 34.1 & 0.616 & - \\
\hline 4 & 18 & 2.59 & 98.9 & $2.00 \mathrm{mM} \mathrm{NaNO}_{3}$ & 2.11 & 68.0 & 27.6 & 0.754 & - \\
\hline 5 & 18 & 5.04 & 841 & - & 2.10 & 18.9 & 65.6 & 6.88 & $\mathrm{AB}_{3}, 64.1^{\dagger}$ \\
\hline 6 & 18 & 7.29 & 841 & $2.25 \mathrm{mM} \mathrm{NaCl}$ & 0 & 0.817 & 16.0 & 64.9 & $A B_{4}, 67.5^{\dagger}$ \\
\hline 7 & 36 & 0.297 & 49.5 & - & 90.4 & 1.92 & 0 & 0 & $A B, 83.2$ \\
\hline 8 & 36 & 3.30 & 49.5 & $3.00 \mathrm{mM} \mathrm{NaCl}$ & 10.1 & 85.7 & 0 & 0 & - \\
\hline
\end{tabular}

${ }^{*}$ The synthetic yield was based on A-NPs (at least 500 counts) in randomly sampled TEM images of product solutions after removal of empty micelles and before the removal of B@PSPAA; the excess B@PSPAA were not included in the calculations. The isolated purity was calculated from randomly sampled TEM images of purified nanoclusters (at least 1,000 counts); both the nanoclusters and the excess B@PSPAA were counted.

$\uparrow$ Purified using the differential centrifugation method, as illustrated in Figure 4a-d

Purified using the $\mathrm{Ag}$ growth/etching method, as illustrated in Figure $4 \mathrm{e}-\mathrm{g}$. 
Mechanistic analysis of the nanoreactions. Interestingly, at low-salt conditions (Table 1, entry 1) some heterodimers AB@ PSPAA (18.2\%) were observed in addition to $\mathrm{AB}_{2} @ P S P A A$ (74.0\%; $\left.d_{\mathrm{A}}=d_{\mathrm{B}}=18 \mathrm{~nm}\right)$. The partial polymer coverage demonstrated that these dimers were not $\mathrm{B}_{2} @ P S P A A$. The fact that the AB nanoclusters only occurred in the low-salt reactions indicated that they probably did not originate from the dissociation of $\mathrm{AB}_{2}$. Instead, they could be considered as an intermediate before the formation of $\mathrm{AB}_{2}$. Should charge repulsion between the B-NPs be a dominant factor in controlling this nanoreaction, larger B-NPs are expected to trap more $\mathrm{AB}$ nanoclusters, owing to their larger size and stronger charge repulsion. As expected, reacting $18 \mathrm{~nm}$ A-NPs with $36 \mathrm{~nm}$ B-NPs under otherwise similar experimental conditions gave heterodimers AB@PSPAA in excellent yield (90.4\%, Figs 2b and 3c). Synthesis of heterodimers is a major challenge in colloidal assembly. Our simple approach has achieved the same level of specificity as those of DNA-based assembly techniques ${ }^{20,30}$. The AB heterodimers could also be categorized as 'Janus' $\mathrm{NPs}^{12,31}$ in that they possess two diametric faces of different chemical functionalities.

By increasing ionic strength in this reaction $([\mathrm{NaCl}]=3.00 \mathrm{mM})$, $\mathrm{AB}_{2} @$ PSPAA (85.7\%, Fig. 3d) resulted instead. These two reactions ruled out electric or magnetic dipoles as the key controlling factors: although it is possible to argue that transient or static dipoles dictate the linear encounter of $\mathrm{AB}$ with a $\mathrm{B}-\mathrm{NP}$ in forming the $\mathrm{AB}_{2}$ nanocluster (Figs $2 \mathrm{c}$ and $3 \mathrm{~d}$ ), the dipoles cannot explain the non-reactivity of $\mathrm{AB}$ at low ionic strength (Fig. $3 \mathrm{c}$, and entry 7 of Table 1). Any charge-dipole or dipole-dipole interaction would have facilitated the reaction of equation (1) rather than impeding it. Alternatively, a

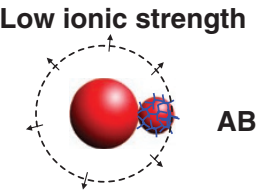

$A B+B \rightarrow A_{2}$

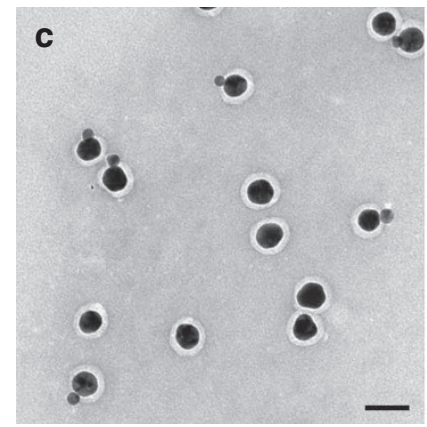

e

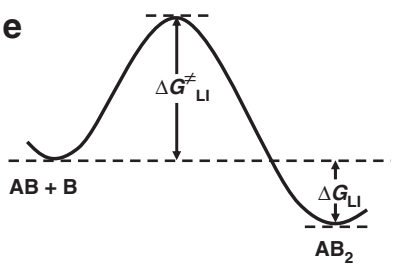

b High ionic strength

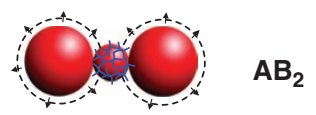

$\mathrm{AB}+\mathrm{B} \longrightarrow \mathrm{AB}_{2}$

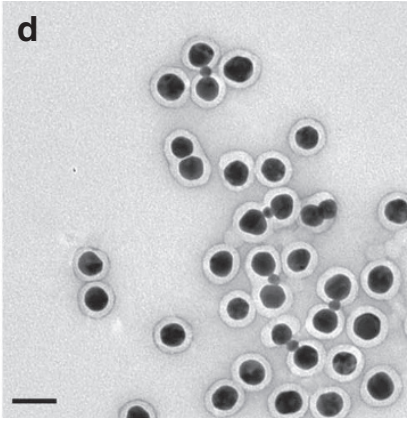

f

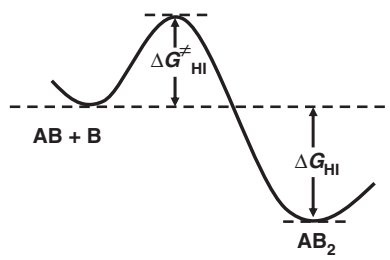

Figure 3 | Understanding the stoichiometry control in the nanoreactions. $(\mathbf{a}, \mathbf{b})$ Schematics showing the syntheses of $A B$ and $A B_{2}$ nanoclusters $\left(d_{A}=18 \mathrm{~nm}, d_{B}=36 \mathrm{~nm}\right)$. Dashed circles with arrows indicate the strength of charge repulsion; larger radius means stronger repulsion. (c, d) TEM images of the $A B$ and $A B_{2}$ samples after PSPAA protection. All scale bars are $50 \mathrm{~nm}$. (e, f) Schematics showing the reaction coordinates of equation (1) at low and high ionic strengths, respectively.

long-range $(>18 \mathrm{~nm})$ charge repulsion between the B-NPs could best explain the non-reactivity of $\mathrm{AB}$; it is also consistent with the formation of $\mathrm{AB}_{2}$ at increased ionic strength. Although it is hard to determine the charge of A-NPs, it is clear that the charge interaction between $\mathrm{A}$ - and $\mathrm{B}-\mathrm{NP}$ has a minor role as $\mathrm{AB}$ clusters readily formed even at lowest ionic strength.

$$
\mathrm{AB}+\mathrm{B} \rightarrow \mathrm{AB}_{2}
$$

To understand how charge interactions affect the nanoreactions, it is critical to determine whether the reactions are thermodynamically or kinetically controlled. In equation (1), the covalent bonding between A- and B-NPs should be the main driving force for the reaction, although other factors, such as charge-charge interactions $\left(V_{\text {elec }}\right)$, Van der Waals interactions, solvophobic effects and possibly also charge-dipole or dipole-dipole interactions, may contribute to the $\Delta G$. On the other hand, the transition state should occur at a point before the chemical bond formation (Fig. $3 \mathrm{e}-\mathrm{f}$ ), where the long-range charge interaction would dominate. Thus, the $V_{\text {elec }}$ has to be overcome before effective collision could take place, making it a major contributor of $\Delta G^{\neq}$. Hence, both $\Delta G$ and $\Delta G^{\neq}$should decrease at high ionic strength owing to weak charge repulsion. This leads to two scenarios that could explain the non-reactivity of $\mathrm{AB}$ at low ionic strength. If the reactions are thermodynamically controlled, the kinetic energy (KE) of the NPs should exceed the activation barrier $\left(\Delta G^{\neq}\right)$, whereas $\Delta \mathrm{G}_{\mathrm{HI}}$ (HI stands for high ionic strength) should be $<0$ at high ionic strength (forward reaction), and $\Delta \mathrm{G}_{\mathrm{LI}}>0$ at low ionic strength (backward reaction). That is, the system was at or close to equilibrium at the end of reaction and the $\mathrm{AB}$ nanoclusters formed because they were the most stable state. In contrast, kinetically controlled reactions would mean that $\mathrm{KE}>\Delta G_{\mathrm{HI}}^{ \pm}$(forward reaction), but $\mathrm{KE}<\Delta G_{\mathrm{LI}}^{\neq}$(no reaction); both $\Delta \mathrm{G}_{\mathrm{LI}}$ and $\Delta \mathrm{G}_{\mathrm{HI}}$ should be $<0$ in this case. Hence, the $\mathrm{AB}$ nanoclusters accumulated, because the KE of the reactants was insufficient to cross the activation barrier to make $\mathrm{AB}_{2}$.

Experiments were carried out to distinguish these two possibilities. After the formation of $\mathrm{AB}_{3}$ clusters $\left(d_{\mathrm{A}}=d_{\mathrm{B}}=18 \mathrm{~nm}\right)$, the colloid was diluted and incubated in an aqueous solution at low ionic strength $(0.297 \mathrm{mM})$ before PSPAA encapsulation. However, few $\mathrm{AB}_{2}$ or $\mathrm{AB}$ clusters were obtained even when the incubation was carried out at elevated temperature or under sonication (Supplementary Fig. S9). Therefore, it is unlikely that the nanoreactions were reversible. Furthermore, the $\mathrm{AB}_{3} @ P S P A A$ in Figure 2h often deviated from perfect triangles and sometimes appeared in $\mathrm{T}$ shape, suggesting the addition of a B-NP to a linear BAB intermediate during its formation. This observation is generally consistent with a non-reversible and kinetically controlled reaction.

The electrostatic repulsion potential $\left(V_{\text {elec }}\right)$ between the B-NPs can be estimated by linear superposition approximation ${ }^{32-34}$ :

$$
\begin{gathered}
V_{\mathrm{elec}} \approx \varepsilon \Psi_{i}^{2} \frac{a_{i}^{2}}{h_{m}+2 a_{i}} \mathrm{e}^{-h_{m} / L e} \\
L_{e}=\kappa^{-1}=\left(\frac{\varepsilon k T}{2 \mathrm{e}^{2} I}\right)^{1 / 2}
\end{gathered}
$$

where $L_{e}=\kappa^{-1}$ is the Debye screening length, calculated from the dielectric constant $\varepsilon$ of the medium, the Boltzmann constant $k$, the temperature $T$, the elementary charge $e$ and the ionic strength $I$ of the electrolyte (equation (3)); $a_{i}$ is the particle radius; $h_{m}$ is the separation distance of the interacting B-NPs; and $\Psi_{i}$ is the surface potential of the particle. Therefore, an increase in the radius of B-NPs results in an increase in $a_{i}^{2} /\left(h_{m}+2 a_{i}\right)$, and thus, a correspondingly larger repulsion potential $V_{\text {elec }}$. Furthermore, as $V_{\text {elec }}$ is proportional to $\exp (-\sqrt{I})$, high ionic strength leads to weak repulsion potential, consistent with the experimental results (Table 1). 
In Figure 2, the initial bonding of A-NP and B-NP is favourable, but further attachment of a B-NP on the resulting $\mathrm{AB}$ cluster has to overcome the repulsion between the B-NPs, which becomes stronger as the $\mathrm{AB}_{n}$ cluster increases in size. At a specific ionic strength, the growth of $\mathrm{AB}_{n}$ stops when the KE of the colliding NPs could not exceed the repulsion potential $V_{\text {elec }}$. High ionic strength lowers the $V_{\text {elec }}$, thus allowing more B-NPs to react with each A-NP (Fig. 2a). Understandably, the size variation among the A-NPs and B-NPs will cause unequal charge repulsions and thus lead to non-uniformity in the stoichiometry of the product nanoclusters. This is in drastic contrast to molecular reactions where all atoms or molecules are perfectly uniform.

Purification. To mimic molecular reactions, the products of the nanoreactions have to be purified by removing the excess B@PSPAA. As the overall densities of $\mathrm{AB}_{n} @ P S P A A(n=2-4)$ are significantly larger than that of B@PSPAA, the former was easily isolated by differential centrifugation ${ }^{24}$. Figure $4 a-d$ shows a typical purification, in which the product solution containing $\mathrm{AB}_{3} @ P S P A A$ was carefully layered on top of a saturated $\mathrm{CsCl}$ solution (no concentration gradient was used). After centrifugation, the B@PSPAA and $\mathrm{AB}_{3} @$ PSPAA were separated into two distinct bands of red and blue colour, respectively. The blue band was enriched in $\mathrm{AB}_{3} @ P S P A A$, and its colour indicated surface plasmon coupling among the AuNPs of $\mathrm{AB}_{3}$. This band was isolated, diluted by aq. $\mathrm{NaOH}(\mathrm{pH}=10)$ and then centrifuged again to remove the excess $\mathrm{CsCl}$. The purity of the resulting $\mathrm{AB}_{2^{-}}, \mathrm{AB}_{3^{-}}$and $\mathrm{AB}_{4} @ \mathrm{PSPAA}$, as based on the surveys of over 1,000 nanoclusters in TEM images, were 87.3, 64.1 and 67.5\%, respectively (Fig. 2g-i, Supplementary Figs S10-S12). Most of the impurities in these samples were $\mathrm{AB}_{n} @$ PSPAA nanoclusters. The non-ideal purities achieved here could be attributed to the imper-

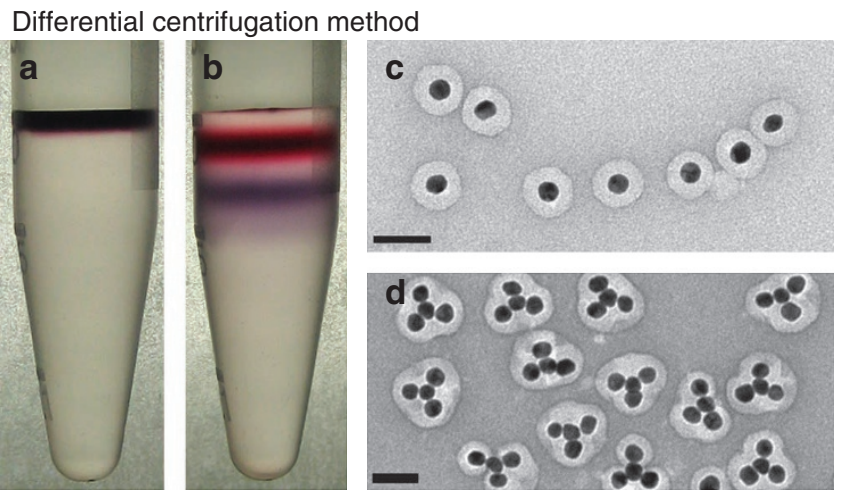

Silver growth/etching method

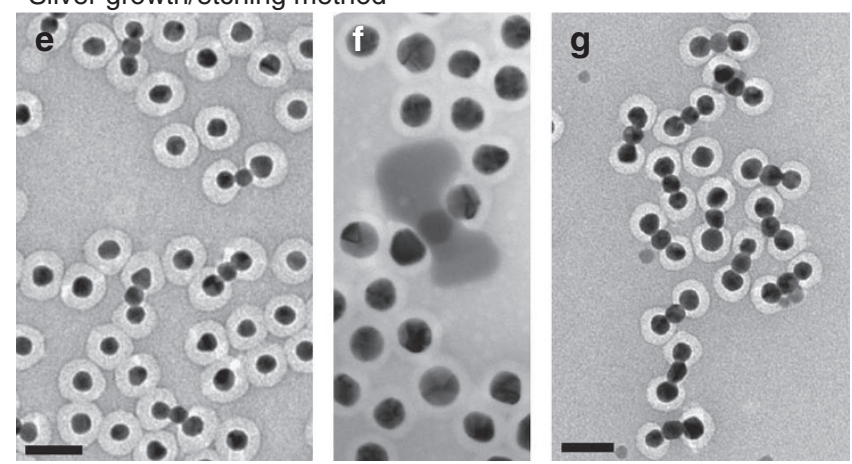

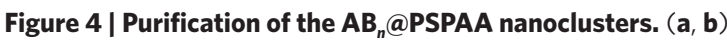

Photographs of a sample solution before and after differential centrifugation. (c, d) TEM images of the separated $B @ P S P A A$ and $A B_{3} @$ PSPAA partitions. (e, f) TEM images of the $A_{2} @ P S P A A$ and $B @ P S P A A$ mixture before and after Ag growth on the A-NPs. (g) TEM image of the sample in $\mathbf{f}$ after centrifugation and etching $\mathrm{Ag}$. All scale bars are $50 \mathrm{~nm}$. fect uniformity in the size of the original AuNPs, which affects both the yield of the nanoreactions and also the efficacy of the purification.

An alternative method was adapted for the separation of $A B$ - and $\mathrm{AB}_{2} @$ PSPAA (Table 1, entry 2 and 7), in which the exposed A-NPs were exploited as seeds for growing metallic Ag (Fig. 4e-f, Supplementary Fig. S13). In contrast, no Ag growth occurred on the PSPAA shells. As a result, the $\mathrm{AB}_{2} @ P S P A A$ grew significantly heavier than the fully encapsulated B@PSPAA, and they could be easily isolated from the latter by centrifugation at low speed in water. Subsequently, the isolated Ag- $\left(\mathrm{AB}_{2} @ \mathrm{PSPAA}\right)$ were washed with $\mathrm{NH}_{3} \cdot \mathrm{H}_{2} \mathrm{O}$ to etch the Ag blocks, and then centrifuged to remove the excess reactants (Fig. 4g). Using this method, $\mathrm{AB}_{2} @$ PSPAA were isolated in $85.9 \%$ purity (Supplementary Fig. S14). This technique was essential for the purification of AB@PSPAA (Fig. 2f, purity 83.2\%, $d_{\mathrm{A}}=18 \mathrm{~nm}$, $d_{\mathrm{B}}=36 \mathrm{~nm}$ ), as there was only a small density difference between the AB- and B@PSPAA (Supplementary Figs. S15 and S16). As such, it is nearly impossible to separate them by the differential centrifugation method. For $\mathrm{AB}_{3}$ - and $\mathrm{AB}_{4} @ P S P A A$, however, the polymer shell tends to enclose the whole nanoclusters without exposing the A-NPs; this prevents Ag attachment and thus renders the purification method ineffective. Hence, the two purification methods are complementary in recovering the nanoreaction products and may allow flexibility in designing future synthetic routes.

So far, in our nanoreactions, the size of the B-NPs is always larger than or equal to the size of A-NPs. This setup effectively used the charge repulsion between B-NPs for the stoichiometry control. Reacting large A-NPs $(36 \mathrm{~nm})$ with small B-NPs $(18 \mathrm{~nm})$ is also feasible (Fig. 5, Supplementary Figs S17 and S18). However, the stoichiometry of the resulting nanoclusters was less uniform, most likely because of the fact that the repulsion between B-NPs was not far enough to affect all neighbours on the large surface of A-NPs. Nevertheless, controlling the ionic strength still by and large controlled the stoichiometry of the $\mathrm{AB}_{n}$ nanoclusters.

\section{Discussion}

We demonstrated a systems approach to colloidal assembly of $\mathrm{Au}$ NPs. By understanding and directing the reaction kinetics, the stoichiometry of the product nanoclusters was controlled simply by modulating the ionic strength of the reaction media. Colloidal synthesis is generally scalable and easy to control; and the polymer a

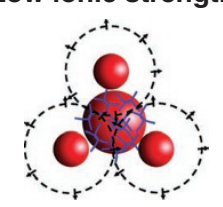

b High ionic strength

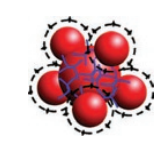

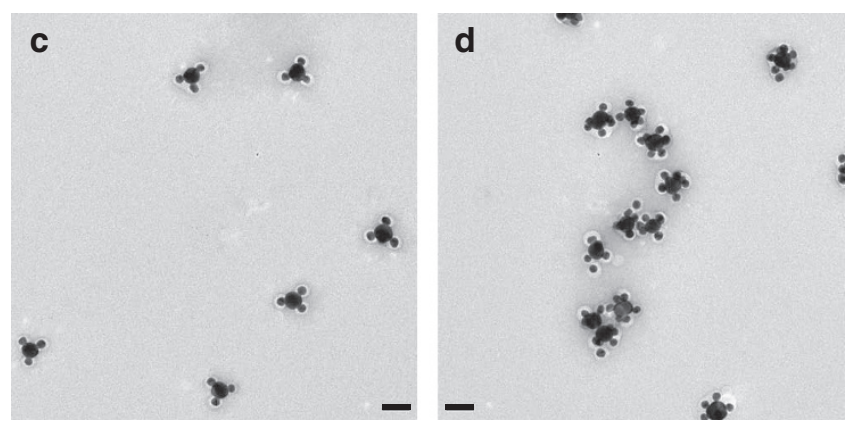

Figure 5 | TEM images of the $A B_{n}$ nanoclusters $\left(d_{A}=36 \mathrm{~nm}, d_{B}=18 \mathrm{~nm}\right)$. (a) Nanoreaction carried out at a low ionic strength $(I=0.594 \mathrm{mM})$; (b) nanoreaction carried out at a high ionic strength $(I=3.30 \mathrm{mM})$. (c, d) TEM images of the resulting nanoclusters of (a) and (b), respectively. All scale bars are $50 \mathrm{~nm}$. 
protection led to the recovery of pure nanoclusters with long-term stability. Hence, this initial platform of NP-based reactions permits further development of synthetic methodologies. In addition, the well-controlled reactions also allow insights into the interactions of NPs. Specifically, charge repulsion has been identified as the major factor in the stoichiometry control in our system, whereas charge or magnetic dipoles were ruled out. The nanoreactions were found to be kinetically controlled: the reaction proceeds at high ionic strength, but strong charge repulsion at low ionic strength gives rise to an insurmountable barrier that diminishes effective collision.

\section{Methods}

Chemicals and instruments. All chemical reagents were obtained from commercial suppliers and used without further purification. Diblock copolymer PSPAA $\left(\mathrm{PS}_{154}-b-\mathrm{PAA}_{60}, M_{\mathrm{n}}=16,000\right.$ for the polystyrene block and $M_{\mathrm{n}}=4,300$ for the poly(acrylic acid) block; $M_{w} / M_{\mathrm{n}}=1.15$ ) was obtained from Polymer Source; Ligand 1 was purchased from Aldrich; Ligand 2 was purchased from Avanti Polar Lipids; DMF (99.8\%) was purchased from Tedia Company. Deionized water (resistance $>18 \mathrm{M} \Omega \mathrm{cm}^{-1}$ ) was used in all of our reactions. All other chemicals were purchased from Sigma-Aldrich. TEM images were collected from a JEM-1400 Transmission Electron Microscope (JEOL) operated at $120 \mathrm{kV}$. UV-visible spectra were collected on a Cary $100 \mathrm{UV}$-Vis spectrophotometer.

Preparation of TEM samples. $\left(\mathrm{NH}_{4}\right)_{6} \mathrm{Mo}_{7} \mathrm{O}_{24}$ was used as a negative stain $(3.4 \mathrm{mM})$ in all TEM images reported in this paper, so that polymer shells appeared white against a dark background. TEM grids were treated by oxygen plasma in a Harrick plasma cleaner/sterilizer for $1 \mathrm{~min}$ to improve the surface hydrophilicity. A sample solution was carefully mixed with stain solution on the surface of a plastic Petridish, forming a small bead; a TEM grid was then floated on the top of the bead with the hydrophilic face contacting the solution. The TEM grid was then carefully picked up by a pair of tweezers, wicked with filter paper to remove excess solution and finally dried in air for $20 \mathrm{~min}$.

Synthesis of AuNPs. Two different size of sodium citrate-stabilized AuNPs ( $18 \mathrm{~nm}$ (ref. 35) and $36 \mathrm{~nm}$ (ref. 36)) were prepared based on the literature reports with small modifications (more details available in Supplementary Information).

Synthesis of A-NPs (1-AuNPs). First, $1.5 \mathrm{ml}$ citrate-AuNPs $\left(d_{\mathrm{AuNP}}=18 \mathrm{~nm}\right)$ was concentrated to $100 \mu \mathrm{l}$ by centrifugation at $13,300 \mathrm{~g}$ for $15 \mathrm{~min}$. Then the AuNPs solution was added dropwise to $300 \mu \mathrm{l}$ of $\mathbf{1}$ in ethanol $\left(4 \mathrm{mg} \mathrm{ml}^{-1}\right)$. The mixture was incubated for $2 \mathrm{~h}$. Then, the A-NPs were purified twice by centrifugation in DMF to remove the excess 1 . These solutions were typically used within $15 \mathrm{~min}$, otherwise partial ligand dissociation can lead to aggregation of the A-NPs.

Preparation of B-NPs solution. B-NPs solution was prepared by concentrating the citrate-AuNPs solution and re-dispersing the NPs in water to remove the excess $\mathrm{Na}_{3}$ citrate from the as-synthesized solution.

Setup of the nanoreactions. The salt concentrations in the B-NPs solution were carefully controlled in the reactions using the synthesis of $\mathrm{AB}_{2}$ nanoclusters as an example here (details for the syntheses of other $\mathrm{AB}_{n}$ nanoclusters can be found in the Supplementary Methods): citrate-AuNPs $\left(3 \mathrm{ml}, d_{\mathrm{AuNP}}=18 \mathrm{~nm}\right)$ were concentrated to $20 \mu \mathrm{l}$ by centrifugation at $13,300 \mathrm{~g}$ for $15 \mathrm{~min}$ and then diluted by adding $180 \mu \mathrm{l} \mathrm{H}$ O. The residue $\left[\mathrm{Na}_{3}\right.$ citrate] was estimated to be $98.9 \mu \mathrm{M}$ and $[\mathrm{B}]=24.3 \mathrm{nM}$ (in number of NPs). All other conditions were the same: the purified A-NPs in DMF $\left(100 \mu \mathrm{l}, d_{\mathrm{ANP}}=18 \mathrm{~nm},[\mathrm{~A}]=3.04 \mathrm{nM}\right)$ were slowly added to an aqueous solution containing large excess of B-NPs $(200 \mu \mathrm{l})$. During the addition, the Eppendorf tube was vortexed to ensure adequate mixing. The reactions are considered to be completed after the mixing; further incubation of the mixture did not alter the results.

Polymer encapsulation of the reaction products. A volume of $740 \mu \mathrm{l} \mathrm{DMF}$ was added to the reaction mixture, which contains $100 \mu \mathrm{l} \mathrm{DMF}$ and $200 \mu \mathrm{l} \mathrm{H}_{2} \mathrm{O}$, followed by $60 \mu \mathrm{PS}_{154} \mathrm{PAA}_{60}$ in DMF $\left(8 \mathrm{mg} \mathrm{ml}^{-1}\right)$ (refs 6, 25). Finally, $40 \mu \mathrm{l}$ of 2 in ethanol $\left(2 \mathrm{mg} \mathrm{ml}^{-1}\right)$ was added. The mixture was heated to $100^{\circ} \mathrm{C}$ for $2 \mathrm{~h}$ and then slowly cooled in the oil bath until it reached room temperature. To isolate the nanoclusters from the excess reactants and empty PSPAA micelles, the product solution was diluted $(200 \mu \mathrm{l}$ diluted by $1,300 \mu \mathrm{laq}$. $\mathrm{NaOH}(\mathrm{pH}=10))$, and then centrifuged at $13,300 \mathrm{~g}$ (or 5,200 $\mathrm{g}$ when $36 \mathrm{~nm} \mathrm{B-NPs}$ were used) for $20 \mathrm{~min}$ to remove the supernatant. The collected purplish-red product at the bottom of centrifuge tubes was diluted by $100 \mu \mathrm{l}$ aq. $\mathrm{NaOH}(\mathrm{pH}=10)$, giving a stable solution that contained mainly $\mathrm{AB}_{n} @ \mathrm{PSPAA}$ and $\mathrm{B} @ \mathrm{PSPAA}$

Purification of $\mathrm{AB}_{n} @$ PSPAA nanoclusters $(\boldsymbol{n}=\mathbf{2}-\mathbf{4})$. In a typical separation, the mixture of $\mathrm{AB}_{n} @ \mathrm{PSPAA}$ and B@PSPAA $(100 \mu \mathrm{l})$ was carefully layered on the top of $1,000 \mu \mathrm{l}$ saturated $\mathrm{CsCl}$ solution in an Eppendorf tube, and the tube was cen- trifuged at 2,300 $\mathrm{g}$ for $25 \mathrm{~min}$ (ref. 24). The resulting solution showed two distinct bands of red and blue colour, separated by a gap. The lower band was extracted, diluted by aq. $\mathrm{NaOH}(\mathrm{pH}=10)$ and then centrifuged again to remove the excess $\mathrm{CsCl}$

Purification of AB $\mathbf{A}_{n} @$ PSPAA nanoclusters $(\boldsymbol{n}=\mathbf{1}, \mathbf{2})$. The collected AB $\mathrm{B}_{2} @ P S P A A$ and B@PSPAA mixture in aq. $\mathrm{NaOH}(\mathrm{pH}=10 ; 100 \mu \mathrm{l})$ was added to $800 \mu$ l sodium citrate $(1.161 \mathrm{mM})$, followed by $400 \mu \mathrm{l} \mathrm{ANO}_{3}(1 \mathrm{mM})$. Then, $400 \mu \mathrm{l}$ ascorbic acid $(2 \mathrm{mM})$ was added and the mixture was incubated for $2 \mathrm{~h}$ on a shaker. The mixture solution was purified three times by centrifugation at $1,400 \mathrm{~g}$ for $15 \mathrm{~min}$ and the supernatant containing the unaffected B@PSPAA were removed. The concentrated brown solution was added to $1,000 \mu \mathrm{N} \mathrm{N}_{3} \cdot \mathrm{H}_{2} \mathrm{O}$ to etch the Ag blocks and the mixture was incubated in an orbital shaker for $12 \mathrm{~h}$ with exposure to air. Finally, the purified $\mathrm{AB}_{2} @$ PSPAA nanoclusters were collected by centrifugation at 2,200g for $15 \mathrm{~min}$. The purification of AB@PSPAA $\left(d_{\mathrm{A}}=18 \mathrm{~nm}, d_{\mathrm{B}}=36 \mathrm{~nm}\right)$ was similar as that for $\mathrm{AB}_{2}$ nanoclusters, except that different centrifugation speeds were used. After Ag growth on the surface of the $\mathrm{AB}$ nanoclusters, the resulting solution was purified three times by centrifugation at $200 \mathrm{~g}$ for $20 \mathrm{~min}$. The etching step was repeated once. Finally, the purified AB@PSPAA were collected by centrifugation at $2,200 \mathrm{~g}$ for $20 \mathrm{~min}$.

\section{References}

1. Lu, X. M., Rycenga, M., Skrabalak, S. E., Wiley, B. \& Xia, Y. N. Chemical synthesis of novel plasmonic nanoparticles. Annu. Rev. Phys. Chem. 60, 167-192 (2009)

2. Grzelczak, M., Perez-Juste, J., Mulvaney, P. \& Liz-Marzan, L. M. Shape control in gold nanoparticle synthesis. Chem. Soc. Rev. 37, 1783-1791 (2008).

3. Peng, X. G. et al. Shape control of CdSe nanocrystals. Nature 404, 59-61 (2000)

4. Murray, C. B., Norris, D. J. \& Bawendi, M. G. Synthesis and characterization of nearly monodisperse $\mathrm{CdE}(\mathrm{E}=\mathrm{S}, \mathrm{Se}, \mathrm{Te})$ semiconductor nanocrystallites. J. Am. Chem. Soc. 115, 8706-8715 (1993).

5. Wang, F. et al. Simultaneous phase and size control of upconversion nanocrystals through lanthanide doping. Nature 463, 1061-1065 (2010).

6. Wang, X. J. et al. Polymer-encapsulated gold-nanoparticle dimers: facile preparation and catalytical application in guided growth of dimeric ZnO-nanowires. Nano Lett. 8, 2643-2647 (2008).

7. Li, W. Y., Camargo, P. H. C., Lu, X. M. \& Xia, Y. N. Dimers of silver nanospheres: facile synthesis and their use as hot spots for Surface-Enhanced Raman Scattering. Nano Lett. 9, 485-490 (2009)

8. Chen, G. et al. Measuring ensemble-averaged Surface-Enhanced Raman Scattering in the hotspots of colloidal nanoparticle dimers and trimers. J. Am. Chem. Soc. 132, 3644-3645 (2010).

9. Dadosh, T. et al. Measurement of the conductance of single conjugated molecules. Nature 436, 677-680 (2005).

10. Brousseau, L. C., Novak, J. P., Marinakos, S. M. \& Feldheim, D. L. Assembly of phenylacetylene-bridged gold nanocluster dimers and trimers. Adv. Mater. 11, 447-449 (1999).

11. Lee, J. H. et al. Site-specific control of distances between gold nanoparticles using phosphorothioate anchors on DNA and a short bifunctional molecular fastener. Angew. Chem. Int. Ed. 46, 9006-9010 (2007).

12. Chen, T., Yang, M. X., Wang, X. J., Tan, L. H. \& Chen, H. Y. Controlled assembly of eccentrically encapsulated gold nanoparticles. J. Am. Chem. Soc. 130, 11858-11859 (2008).

13. Alivisatos, A. P. et al. Organization of 'nanocrystal molecules' using DNA. Nature 382, 609-611 (1996)

14. Sung, K. M., Mosley, D. W., Peelle, B. R., Zhang, S. G. \& Jacobson, J. M. Synthesis of monofunctionalized gold nanoparticles by Fmoc solid-phase reactions. J. Am. Chem. Soc. 126, 5064-5065 (2004).

15. DeVries, G. A. et al. Divalent metal nanoparticles. Science 315, 358-361 (2007)

16. Nakata, K., Hu, Y., Uzun, O., Bakr, O. \& Stellacci, F. Chains of superparamagnetic nanoplarticles. Adv. Mater. 20, 4294-4299 (2008).

17. Loweth, C. J., Caldwell, W. B., Peng, X. G., Alivisatos, A. P. \& Schultz, P. G. DNA-based assembly of gold nanocrystals. Angew. Chem. Int. Ed. 38, 1808-1812 (1999).

18. Xu, X. Y., Rosi, N. L., Wang, Y. H., Huo, F. W. \& Mirkin, C. A. Asymmetric functionalization of gold nanoparticles with oligonucleotides. J. Am. Chem. Soc. 128, 9286-9287 (2006).

19. Sardar, R., Heap, T. B. \& Shumaker-Parry, J. S. Versatile solid phase synthesis of gold nanoparticle dimers using an asymmetric functionalization approach. J. Am. Chem. Soc. 129, 5356-5357 (2007).

20. Maye, M. M., Nykypanchuk, D., Cuisinier, M., van der Lelie, D. \& Gang, O. Stepwise surface encoding for high-throughput assembly of nanoclusters. Nat. Mater. 8, 388-391 (2009).

21. Mastroianni, A. J., Claridge, S. A. \& Alivisatos, A. P. Pyramidal and chiral groupings of gold nanocrystals assembled using DNA scaffolds. J. Am. Chem. Soc. 131, 8455-8459 (2009).

22. Maneeprakorn, W., Malik, M. A. \& O’Brien, P. Developing chemical strategies for the assembly of nanoparticles into mesoscopic objects. J. Am. Chem. Soc. 132, 1780-1781 (2010).

23. Yang, M. X. et al. Development of polymer-encapsulated metal nanopartictes as Surface-Enhanced Raman Scattering probes. Small 5, 198-202 (2009). 
24. Chen, G. et al. High-purity separation of gold nanoparticle dimers and trimers. J. Am. Chem. Soc. 131, 4218-4219 (2009).

25. Chen, H. Y. et al. Encapsulation of single small gold nanoparticles by diblock copolymers. ChemPhysChem. 9, 388-392 (2008).

26. Kang, Y. J. \& Taton, T. A. Core/shell gold nanoparticles by self-assembly and crosslinking of micellar, block-copolymer shells. Angew. Chem. Int. Ed. 44, 409-412 (2005).

27. Crespo, P. et al. Permanent magnetism, magnetic anisotropy, and hysteresis of thiol-capped gold nanoparticles. Phys. Rev. Lett. 93, 087204 (2004).

28. Zhang, H. \& Wang, D. Y. Controlling the growth of charged-nanoparticle chains through interparticle electrostatic repulsion. Angew. Chem. Int. Ed. 47, 3984-3987 (2008).

29. Xing, S. X. et al. Highly controlled core/shell structures: tunable conductive polymer shells on gold nanoparticles and nanochains. J. Mater. Chem. 19, 3286-3291 (2009).

30. Fu, A. H. et al. Discrete nanostructures of quantum dots/Au with DNA. J. Am. Chem. Soc. 126, 10832-10833 (2004).

31. Walther, A. \& Muller, A. H. E. Janus particles. Soft Matter 4, 663-668 (2008).

32. Bell, G. M., Levine, S. \& McCartney, L. N. Approximate methods of determining the double-layer free energy of interaction between two charged colloidal spheres. J. Colloid Interface Sci. 33, 335-359 (1970).

33. Aizenberg, J., Braun, P. V. \& Wiltzius, P. Patterned colloidal deposition controlled by electrostatic and capillary forces. Phys. Rev. Lett. 84, 2997-3000 (2000).

34. Adamczyk, Z., Jaszczolt, K., Michna, A., Zembala, M. \& Barbasz, J. in Colloid Stability: The Role of Surface Forces Part II, Vol. 2. Colloids and Interface Science Series (ed. Tadros, T. F.) Ch. 9, 250-258 (Wiley, 2007).

35. Frens, G. Controlled nucleation for regulation of particle-size in monodisperse gold suspensions. Nature Phys. Sci. 241, 20-22 (1973).
36. Brown, K. R., Walter, D. G. \& Natan, M. J. Seeding of colloidal Au nanoparticle solutions. 2. Improved control of particle size and shape. Chem. Mater. 12, 306-313 (2000).

\section{Acknowledgments}

We thank Ministry of Education, Singapore (ARC 27/07 and 13/09) for financial support. S.L. thanks the support in computational facilities from theoretical groups at the Northwestern University.

\section{Author contributions}

Y.W. and H.C. designed and planned this project. Y.W. was responsible for the synthesis of nanoreaction products and characterization. G.C. helped to purify the large $\mathrm{AB}_{n} @$ PSPAA $(n \geq 2)$ nanoreaction products. S.X., L.H.T. and Y.F. supported the purification of $\mathrm{AB} @ \mathrm{QPSPAA}(n \leq 2)$ nanoreaction products. M.Y., G.S. F.W. and X.L. performed the theoretical analysis of charge repulsion. S.L. calculated the extinction spectra of $\mathrm{AB}_{n}$ nanoclusters. All authors participated in the mechanistic discussions and contributed to the writing and revising of the paper.

\section{Additional information}

Supplementary Information accompanies this paper on http://www.nature.com/ naturecommunications.

Competing financial interests: The authors declare no competing financial interests. Reprints and permission information is available online at http://npg.nature.com/ reprintsandpermissions/

How to cite this article: Wang, Y. et al. A systems approach towards the stoichiometry-controlled hetero-assembly of nanoparticles. Nat. Commun. 1:87 doi: $10.1038 /$ ncomms1089 (2010) 